\title{
Análise da Revisão Cochrane: O Tamanho das Porções, das Embalagens, dos Pratos e dos Copos para Alterar a Seleção e o Consumo de Alimentos, Bebidas Alcoólicas e Tabaco. Cochrane Database Syst Rev. 2015;09: CD011045.
}

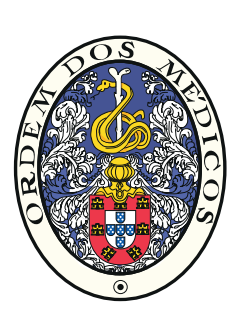

\section{Analysis of the Cochrane Review: Portion, Package or Tableware Size for Changing Selection and Consumption of Food, Alcohol and Tobacco. Cochrane Database Syst Rev. 2015;09: CD011045.}

\author{
Gonçalo S. DUARTEE ${ }^{1,2,3}$, Filipe BROGUEIRA RODRIGUES ${ }^{1,2,4}$, João COSTA ${ }^{1,2,3}$, António VAZ-CARNEIRO $\triangle^{3,5}$ \\ Acta Med Port 2016 Jul-Aug;29(7-8):421-424 - http://dx.doi.org/10.20344/amp.7854
}

\section{RESUMO}

Algumas das principais causas de mortalidade e morbilidade nos países desenvolvidos - como as doenças cardiovasculares, neurológicas e oncológicas - são dependentes de fatores de risco modificáveis. Estratégias de prevenção primordial/primária que alterem o meio ambiente em que vivemos podem ter impacto nestes fatores de risco. Os autores desta revisão sistemática Cochrane procuraram evidência em ensaios clínicos controlados e randomizados para perceber se o tamanho das porções, embalagens, pratos e copos tinham influência no consumo e seleção de alimentos, bebidas alcoólicas e não alcoólicas e produtos derivados do tabaco, tendo concluído que as escolhas de tamanho maior estão associadas a um incremento na seleção e consumo de alimentos, bebidas não alcoólicas e cigarros.

Palavras-chave: Alimentação; Consumo de Bebidas Alcoólicas; Embalagem de Produtos; Ingestão de Alimentos; Preferências Alimentares; Revisão Sistemática; Tabagismo; Tamanho da Porção; Utensílios de Alimentação e Culinária.

\section{ABSTRACT}

Some of the main causes of mortality and morbidity among the developed countries - such as the cardiovascular, neurological and oncologic diseases - are deeply associated with modifiable risk factors. Primordial/primary prevention strategies that alter our environment can have an impact on these risk factors. The authors of this Cochrane systematic review sought evidence from randomized controlled trials to study the effect of the size of portions, packages, dishes and cups, as well as their respective formats, on the consumption and selection of food, alcoholic and non-alcoholic beverages and tobacco products. Overall, this review concludes that the choice of larger portions results in an increased consumption in food, non-alcoholic beverages and tobacco.

Keywords: Alcohol Drinking; Cooking and Eating Utensils; Eating; Food Preferences; Portion Size; Product Packaging; Randomized Controlled Trials as Topic; Smoking.

\section{QUESTÃO CLÍNICA}

Será que o tamanho e formato das porções, das embalagens, dos pratos e dos copos têm influência na escolha e consumo de alimentos, bebidas não alcoólicas, bebidas alcoólicas e produtos derivados da planta do tabaco por parte de adultos e crianças? ${ }^{1}$

\section{OBJECTIVOS}

Avaliar os efeitos de intervenções envolvendo a exposição a diferentes tamanhos e formatos de porções, embalagens, pratos e copos no consumo de alimentos, bebidas não alcoólicas e alcoólicas, e tabaco, em adultos e crianças.

\section{METODOLOGIA}

Revisão sistemática de ensaios clínicos aleatorizados e controlados comparando os efeitos da exposição a diferentes tamanhos das porções, das embalagens, e dos talheres na seleção e consumo de alimentos, bebidas alcoólicas e tabaco, em adultos e crianças. Foram excluídos estudos observacionais. Para identificar os ensaios clínicos relevantes foram pesquisadas as seguintes bases de dados até janeiro de 2015: Cochrane Central Register of Controlled Trials; MEDLINE; EMBASE; PsycINFO; ProQuest; Web of Knowledge; EPPI Centre; Open Grey; ClinicalTrials.gov; e ICTRP. Dois investigadores independentes selecionaram os estudos, extraíram os dados e avaliaram a qualidade metodológica. As principais medidas de resultado foram: a seleção e o consumo dos produtos.

\footnotetext{
1. Laboratório de Farmacologia Clínica e Terapêutica. Faculdade de Medicina. Universidade de Lisboa. Lisboa. Portugal.

2. Clinical Pharmacology Unit. Instituto de Medicina Molecular. Lisboa. Portugal.

3. Centro de Estudos de Medicina Baseada na Evidência. Faculdade de Medicina. Universidade de Lisboa. Lisboa. Portugal.

4. Huntington's Disease Centre. Department of Neurodegenerative Disease. Institute of Neurology. University College London. London. United Kingdom.

5. Centro Colaborador Português da Rede Cochrane Ameroamerica. Lisboa. Portugal.

$\triangle$ Autor correspondente: António Vaz-Carneiro. avc@medicina.ulisboa.pt

Recebido: 16 de maio de 2016 - Aceite: 16 de maio de 2016 | Copyright $\odot$ Ordem dos Médicos 2016
} 


\section{RESULTADOS}

Ao todo foram incluídos 72 ensaios clínicos aleatorizados e controlados, publicados entre 1978 e 2012, com um risco de viés elevado a indeterminado (Tabela 1). Noventa e seis por cento dos estudos avaliou intervenções relacio- nadas com a alimentação, quatro por cento com tabaco e nenhum com bebidas alcoólicas. Quarenta e nove por cento estudou o tamanho das porções, $21 \%$ estudou o tamanho e forma dos pratos e copos, e $14 \%$ estudou o tamanho das embalagens. A grande maioria - $76 \%$ - dos estudos

Tabela 1 - Sumário de resultados, adotada da versão original

\begin{tabular}{|c|c|c|c|c|c|}
\hline \multicolumn{6}{|c|}{$\begin{array}{l}\text { Pacientes ou população: Crianças e adultos } \\
\text { Contexto: Países desenvolvidos } \\
\text { Intervenção: Porções, embalagens, pratos e copos grandes } \\
\text { Comparação: Porções, embalagens, pratos e copos pequenos }\end{array}$} \\
\hline \multirow[t]{3}{*}{ Resultados } & \multicolumn{2}{|c|}{ Riscos comparativos ilustrativos * (IC 95\%) } & \multirow{3}{*}{$\begin{array}{l}\text { Efeito relativo } \\
\text { (IC 95\%) }\end{array}$} & \multirow{3}{*}{$\begin{array}{l}\text { Número de } \\
\text { participantes } \\
\text { (estudos) }\end{array}$} & \multirow{3}{*}{$\begin{array}{l}\text { Qualidade da } \\
\text { evidência } \\
\text { (GRADE) }^{* *}\end{array}$} \\
\hline & Risco assumido & Risco correspondente & & & \\
\hline & $\begin{array}{c}\text { Porções, embalagens, pratos e } \\
\text { copos pequenos }\end{array}$ & $\begin{array}{l}\text { Porções, embalagens, pratos e } \\
\text { copos grandes }\end{array}$ & & & \\
\hline
\end{tabular}

Alimentos:

Seleção O consumo energético médio diá- O consumo energético médio diário A seleção média no grupo 1164 Moderada $^{\mathrm{b}}$ rio de alimentos numa população de alimentos seria 209 kcal (12,4\%) intervenção foi $\mathbf{0 , 4 2}$ des- (10 estudos) representativa de crianças e adul- superior na mesma população. vios padrões superior (de tos no Reino Unido é $1689 \mathrm{kcal}^{\mathrm{a}}$ 0,24 a 0,59 )

Consumo O consumo energético médio diá- O consumo energético médio diário O consumo médio no gru- 6603
rio de alimentos numa população de alimentos seria 189 kcal (11,2\%) po intervenção foi $\mathbf{0 , 3 8}$ (58 estudos) representativa de crianças e adul- superior na mesma população. desvios padrões superior tos no Reino Unido é $1689 \mathrm{kcal}^{\mathrm{a}}$ (de 0,29 a 0,46$)$

Bebidas não alcoólicas:

Seleção

O consumo energético médio de O consumo energético médio por re- O consumo médio no gru- 232

Baixa $^{\text {b,d }}$ bebidas não alcoólicas por refei- feição seria $242(\mathbf{1 0 3 , 4} \%)$ superior na po intervenção foi 1,47 (3 estudos) ção numa população represen- mesma população. desvios padrões superior tativa de crianças e adultos no (de 0,52 a 2,43$)$ Reino Unido é $234 \mathrm{~g}^{\mathrm{a}}$

Consumo O consumo energético médio de $\mathrm{O}$ consumo energético médio por re- $\mathrm{O}$ consumo médio no gru- 50 bebidas não alcoólicas por refei- feição seria 195 (79,6\%) superior na po intervenção foi 1,17 (1 estudo) ção numa população represen- mesma população. desvios padrões superior tativa de crianças e adultos no (de 0,57 a 1,78$)$ Reino Unido é $234 \mathrm{~g}^{\mathrm{a}}$

\section{Produtos derivados do tabaco:}

Seleção Sem evidência disponível $\quad$ - 0

Consumo

O consumo médio de produtos O consumo médio acrescido de pro- O consumo médio no gru- 108 (0 estudos) derivados do tabaco numa popu- dutos derivados do tabaco seria 2 po intervenção foi $\mathbf{0 , 2 5}$ (3 estudos) lação representativa de adultos (1 a 5) cigarros superior na mesma desvios padrões superior no Reino Unido é de 13 cigarros $^{c}$ população $\quad($ de $\mathbf{0 , 1 4}$ a $\mathbf{0 , 6 5})$

Bebidas alcoólicas:

\begin{tabular}{|c|c|c|c|c|}
\hline Seleção & Sem evidência disponível & - & $\begin{array}{l}0 \\
\text { (0 estudos) }\end{array}$ & - \\
\hline Consumo & Sem evidência disponível & - & $\begin{array}{l}0 \\
\text { (0 estudos) }\end{array}$ & - \\
\hline
\end{tabular}

IC: Intervalo de confiança.

* O risco assumido define-se como a mediana do risco dos grupos de controlo. O risco correspondente baseia-se no risco assumido no grupo de comparação e o efeito relativo da intervenção.

** Sistema GRADE:

Qualidade alta: investigação ulterior é altamente improvável que altere a confiança na estimativa do efeito

Qualidade moderada: investigação ulterior tem provável impacto na confiança na estimativa do efeito e pode mesmo alterá-la

Qualidade baixa: investigação ulterior tem alta probabilidade de alterar o impacto da confiança na estimativa do efeito e de alterar a própria estimativa do efeito

Qualidade muito baixa: grande incerteza na estimativa do resultado.

a Estimativas baseadas no UK National Diet and Nutrition Survey (National Centre for Social Research 2012).

b O risco de viés dos estudos incluídos (alto a indeterminado) motivou a subclassificação da evidência.

c Estimativas baseadas no UK Opinions and Lifestyle Survey (Office for National Statistics 2012).

${ }^{d}$ A imprecisão dos estudos incluídos motivou a subclassificação da evidência.

${ }^{e}$ As limitações do estudo incluído (alto viés metodológico) motivou a subclassificação da evidência em dois patamares. 
avaliou adultos.

Efeito do tamanho na seleção (10 estudos, 1164 participantes): observou-se que nos adultos, mas não nas crianças, a exposição a porções, pratos e copos de maiores dimensões aumentava a quantidade de alimentos selecionados (diferença médica standard [SMD] de 0,38; IC 95\%: 0,29 a 0,46) quando comparado com elementos de menores dimensões.

Efeito da forma na seleção (3 estudos, 232 participantes): observou-se que a utilização de garrafas ou copos curtos e largos em comparação com garrafas ou copos longos e estreitos favorece a seleção de maiores quantidades de alimentos e bebidas não alcoólicas (SMD de 1,47; IC 95\%: 0,52 a 2,43), quer em adultos, quer em crianças.

Efeito do tamanho no consumo: o grupo ao qual foram oferecidas porções, embalagens ou pratos ou copos maiores (58 estudos, 6603 participantes), consumiu mais comida e bebidas não-alcoólicas (SMD de 0,38; IC 95\%: 0,29 a 0,46). O mesmo fenómeno não foi observado quando se compararam (3 estudos, 108 participantes) cigarros curtos com cigarros compridos no consumo total de tabaco (SMD de 0,25; IC95\%: -0,14 a 0,659).

Efeito de forma no consumo (1 estudo, 50 participantes): os participantes beberam mais água quando comparadas garrafas curtas e largas com garrafas longas e esguias (SMD de 0,42; IC 95\%: 0,24 a 0,59).

\section{CONCLUSÕES}

Esta revisão sistemática e meta-análise indicam - apesar de utilizar informação de qualidade metodológica baixa a indeterminada - que a população em geral tende a consumir e selecionar maiores quantidades de alimentos quando estes são oferecidos em porções, embalagens, ou pratos maiores, ou no caso especifico das bebidas não alcoólicas, em recipientes curtos e largos. Não foi possível inferir conclusões robustas relativamente ao consumo e seleção de bebidas alcoólicas ou tabaco por falta de evidência.

\section{COMENTÁRIO}

A obesidade, o consumo de álcool e o tabagismo são importantes fatores de risco modificáveis para múltiplas doenças cardiovasculares, neurológicas, respiratórias, endocrinologias e oncológicas. ${ }^{2}$ Estas doenças acarretam, em geral, elevada morbilidade, mortalidade e perda de anos de vida úteis. ${ }^{3}$ A procura de estratégias que modifiquem tais fatores de risco é essencial para melhorar a saúde pública.

O meio físico em que o ser humano se insere tem uma importante influência nas suas atitudes, ${ }^{4}$ e a modificação desse ambiente é uma oportunidade para promover melhores comportamentos relacionados com a saúde.

Com esta revisão sistemática, Hollands GJ et al ${ }^{1}$ demonstraram que existe evidência que suporte que a altera- ção do ambiente tem potencial para alterar comportamentos alimentares e exercer um efeito benéfico a nível dos fatores de risco acima referidos.

Uma limitação inerente aos estudos que visam estudar o efeito do ambiente no comportamento humano é o possível controlo sobre o paradigma experimental, muito diferente daquilo que acontece quando se estudam intervenções terapêuticas farmacológicas. As ferramentas desenvolvidas pela Cochrane ${ }^{5}$ para avaliar ensaios clínicos foram conceptualizadas a pensar neste último tipo de estudo sendo por isso expectável que os estudos avaliados por esta revisão jamais consigam atingir valores de qualidade metodológica tão elevados quanto desejável. Este fato não deve ser visto com insegurança ou como fator limitante das conclusões geradas, mas sim como inerente ao modelo criado pela Cochrane.

Infelizmente não existe evidência para o consumo de bebidas alcoólicas, e a evidência para os produtos derivados da planta tabaco é ainda escassa, sendo necessários estudos para informar os clínicos e decisores políticos sobre a eficácia de semelhantes intervenções em tais campos. Não se sabe se este outcome comportamental irá surtir um efeito a nível dos outcomes de saúde finais, isto é, na obesidade, o consumo de álcool e o tabagismo. E ainda, pelas características dos estudos analisados, não se sabe se este efeito se manterá em relação a porções, embalagens ou pratos cada vez mais pequenos.

\section{IMPLICAÇÕES PARA A PRÁTICA CLÍNICA}

- Por inexistência de evidência, não é possível tecer considerações relativamente ao impacto do tamanho e forma de apresentação das bebidas alcoólicas e produtos com tabaco ou derivados.

- A utilização de porções, embalagens ou pratos maiores está associada ao consumo de maiores quantidades de alimentos e o mesmo se verifica para bebidas não alcoólicas com copos e garrafas curtos e largos. Isto sugere que a utilização de porções, embalagens ou pratos de menores dimensões estão associados à diminuição da quantidade de alimentos consumidos e, do mesmo modo, copos e garrafas estreitos e longos associados à diminuição do consumo de bebidas não alcoólicas.

- Neste sentido, os profissionais de saúde podem sugerir tais estratégias como parte da componente educacional individual da prevenção dos fatores de risco cardiovasculares, e os decisores políticos optar por implementar políticas de acordo com este modelo em estabelecimentos públicos como escolas e cantinas e na redação da legislação reguladora do setor da restauração e da indústria alimentar.

2. Alwan A. Global status report on noncommunicable diseases 2010. Geneva: World Health Organization; 2011.

3. World Health Organization. World Health Statistics 2014. Geneva: WHO; 2014. 
4. Hollands GJ, Shemilt I, Marteau TM, Jebb SA, Kelly MP, Nakamura R, et al. Altering choice architecture to change population health behaviour: a large-scale conceptual and empirical scoping review of interventions within micro-environments. 2013.

5. Higgins JP, Green S. Cochrane handbook for systematic reviews of interventions: Wiley Online Library; 2008.



